\title{
Modelación matemática del secado convencional de madera
}

\section{Mathematical modeling of the conventional drying of wood}

\author{
Sadoth Sandoval-Torres*
}

\begin{abstract}
RESUMEN
La operación de secado de madera es una etapa crucial dentro del procesamiento de productos de madera. Durante el secado se generan algunos defectos debido a la distribución variable del contenido de humedad y de la temperatura al interior del material. En el secado industrial de madera, los problemas de optimización de los procesos deben ser fundamentados en los diferentes fenómenos de transporte que controlan la transferencia de masa y calor en el sólido. Por sus propiedades físicas, las maderas duras adquieren gran importancia como materia prima, pues su dureza, color, estabilidad dimensional y alta resistencia a la degradación causada por el medio ambiente, les dan un valor comercial importante. En los últimos años el modelamiento de los procesos de secado ha sido una herramienta eficaz para la optimización de tales operaciones; estos modelos asocian balances de energía, de masa y de momento, que pueden ser expresados como ecuaciones diferenciales que son resueltas numéricamente para obtener soluciones específicas en cada problema. En este trabajo se explican los mecanismos de transferencia de masa y calor presentes en el secado convencional, se escriben las ecuaciones de transporte de masa y calor, y por último se muestran los perfiles de humedad y temperatura para el encino europeo Quercus pedonculae, sometido a secado convencional.
\end{abstract}

PALABRAS CLAVE:

Física de Secado, Transferencia de masa y calor, Madera latifoliada.

\begin{abstract}
The kiln-drying of lumber is a very important stage into wood-based products processing. During drying process, some defects are produced due to the variable moisture and heat distribution inside of material. In order to optimize the kiln-drying of lumber is important to take into account the transport phenomena controlling the heat and mass transfer in the solid. Due to its physical properties, the hardwoods are a very important raw material, since its hardness, colour, dimensional stability and high resistance to weathering give them a very important commercial value. At the last years, the drying process modelling has been an effective tool to optimize such operations; these models associates energy, mass and momentum balances, which can be expressed as differential equations that are numerically solved to obtain particularly solution for each problem. In this work, the heat and mass transfer mechanisms presents in kiln-drying of lumber are explained, heat and mass transport equations are written, and finally moisture and temperature profiles for European Oakwood Quercus pedoncula during kiln-drying are presented.
\end{abstract}

KEY WORDS:

Physics of Drying, Heat and Mass transfer, Hardwood

Chem.Eng. and PhD-Student, CONACYT Fellow in France. University of Science and Technology Bordeaux 1. Ce: ssandova@u-bordeaux1.fr 


\section{INTRODUCCIÓN}

El principal objetivo del secado industrial de madera es acelerar los mecanismos de transferencia de masa, optimizar el consumo energético y, principalmente obtener un producto con mejores atributos de calidad. El secado es una operación unitaria de uso frecuente en varias ramas de la industria. Durante una operación de secado, las maderas experimentan cambios físicos y químicos (Keey et al. 2000), los cuales afectarán su calidad al final del secado, es por eso que el estudio de la transferencia de masa y calor en materiales biológicos adquiere gran importancia. De acuerdo a Martinovic et al. (2001), el secado es un paso muy importante dentro del proceso de manufactura de productos de madera, durante el cual se desarrolla una distribución de humedad y temperatura no uniforme en el material, que se refleja en muchas ocasiones en la generación de defectos de secado. Los fundamentos de los fenómenos de transporte que son aplicados al secado de madera pueden ser consultados en referencias como: Middleman (1997), Incropera et al (2006), Geankoplis (2003), Majumdar (2005) y Bird et al (2006).

Los modelos matemáticos aplicados al secado de materiales están divididos principalmente en tres grupos: 1) modelos empíricos aplicados a procesos específicos, 2) modelos difusivos de transferencia de masa y calor que llevan a ecuaciones simultáneas, y 3) modelos conocidos como comprensivos, los cuales involucran ecuaciones diferenciales de transporte de masa, calor y momentum con fundamentos termodinámicos; estos modelos son conocidos también como mecanísticos. En la actualidad el aporte científico del modelamiento matemático de los procesos de secado es de gran interés por su aplicación industrial, de ahí el interés de diversos centros de investi- gación por la simulación numérica de estas operaciones, la cual es una tarea de gran complejidad numérica y matemática, como puede ser constatado en trabajos como el de A. Esfahanian (1999), Turner y Perré (2001) y Perré y Turner (2002) entre otros.

\section{OBJETIVOS}

1. Escribir las ecuaciones y explicar los mecanismos de transferencia de masa y calor presentes en el secado de la madera.

2. Describir el secado de la madera como una operación unitaria fundamentada en los fenómenos de transporte aplicados a un medio poroso.

3. Presentar un modelo matemático mecanístico para el secado de madera y resolverlo numéricamente.

\section{Materiales porosos y materiales capilaro-porosos}

La distinción entre materiales porosos y materiales capilaro-porosos está basada en la presencia y tamaño de los poros. Los materiales porosos son definidos como aquellos que tienen un diámetro de poro mayor o igual a $10^{-7} \mathrm{~m}$, y los materiales capilaro-porosos tienen un diámetro menor a $10^{-7} \mathrm{~m}$. El transporte de humedad en los materiales capilaro-porosos es un fenómeno difícil de modelisar, pues además de la difusión molecular, el transporte de agua puede ser debido también a la difusión de vapor, difusión superficial, difusión knudsen, flujo capilar, flujo hidrodinámico entre otros mecanismos.

En materiales no higroscópicos, los poros están llenos de agua si el material está saturado y con aire si ellos están completamente secos, estos materiales no se contraen. Por otra parte, en el caso de 
materiales higroscópicos habrá un momento en el cual la presión de vapor de agua será importante, pues llega a ser función del contenido de humedad y de la temperatura del sistema. La madera pertenece al grupo considerado como capilaroporoso, el cual es higroscópico. En los materiales capilaro-porosos, los poros pueden estar comunicados, mientras que en los materiales porosos no necesariamente. Asimismo, la higroscopicidad en la madera, además de la porosidad, también es generada significativamente por su composición química, específicamente por la presencia de radicales $\mathrm{OH}$.

En el presente escrito, los valores y elementos de análisis están orientados al encino europeo la cual es una madera dura. De acuerdo a Williamson (2001), las maderas duras proceden de árboles de hoja ancha, pertenecientes a las angiospermas o conocidas como latifoliadas, y las maderas suaves a las procedentes de árboles con hojas aciculares, pertenecientes a las gimnospermas o confieras. Las maderas en forma general presentas dos secciones importantes, desde el punto de vista estructural: una región conocida como albura y otra conocida como duramen. En la albura se llevará a cabo el transporte de nutrientes para el crecimiento del árbol, con el tiempo la porción central del tronco podrá colectar los excesos en nutrientes, los cuales metaboliza para formar extractivos que otorgarán un color más intenso al duramen. La madera es un material poroso, biológico, anisotrópico y heterogéneo (Truscott y Turner; 2006), el cual posee una estructura fibrosa y celular muy compleja.

\section{Modelos difusivos}

En este trabajo sólo se describen los modelos difusivo y comprensivo para el secado de madera, pues los modelos empíricos no son de interés para este escrito. Los modelos de ecuaciones dife- renciales (MEF) de secado son basados en las leyes de transferencia de masa y calor, los cuales son resueltos con diferentes métodos numéricos, y llevan a soluciones particulares de cada problema.

El flujo de contenido de humedad en el producto ocurre por difusión y es gobernado por un coeficiente de difusión efectivo, siguiendo la ley de Fick, entonces el balance de masa en la tabla sera:

$$
D_{\text {humedad }} \nabla \cdot\left(\nabla \rho_{\text {madera }} W\right)=\frac{\partial\left(\rho_{\text {madera }} W\right)}{\partial t}
$$

La ecuación anterior establece que la rapidez de cambio en el contenido de humedad $W$ del material, es igual a la difusion de agua debido al gradiente de contenido de humedad interno de la tabla

$$
\nabla \rho_{\text {madera }} W
$$

El producto se contrae durante el secado, sin embargo, los efectos de encogimiento, densidad y propiedades térmicas del producto son normalmente despreciados.

En la actualidad, para fines prácticos y de modelación matemática, la cantidad de agua presente en la madera es clasificada como agua libre, agua ligada y vapor de agua (Siau, 1984). El transporte de estos tres tipos de humedad son combinados para obtener un flux de agua simple $j$. Entonces la ecuación puede ser escrita como:

$$
j=D_{\text {humedad }} \nabla \cdot\left(\nabla \rho_{\text {madera }} W\right)
$$

El flux másico de humedad $j$ es proporcional a la difusividad del agua en el producto, $D_{\text {humedad }}$ 


$$
\rho_{\text {madera }}\left(\frac{\partial C p_{\text {madera }} T_{\text {madera }}}{\partial t}\right)+C p_{\text {madera }} T_{\text {madera }} \frac{\partial \rho_{\text {madera }}}{\partial t}=\nabla \cdot\left(k \cdot \nabla T_{\text {madera }}\right)-C p_{\text {agua }} j\left(\nabla T_{\text {madera }}\right)
$$

\section{Ecuaciones para la transferencia de calor}

La transferencia de calor al interior de una tabla de madera es dada por conducción, teniendo como parámetro motor el gradiente de temperatura que se desarrolla a lo largo del espesor de la tabla. La ecuación que describe el transporte de enegía en la tabla es escrita como se indica en la ecuación 3.

En esa ecuación, el cambio de la energía interna en un volumen de control en un lapso de tiempo es representado por los dos términos a la izquierda de la ecuación. Este balance de energía establece que la rapidez de cambio en la energía interna es igual a la diferencia entre la rapidez neta de calor transportado hacia adentro del volumen de control debido a la conducción,

$$
\nabla \cdot\left(k_{\text {madera }} \nabla T_{\text {madera }}\right)
$$

y la rapidez a la cual el calor es transportado fuera del volumen de control debido al flujo másico, $C p_{\text {agua }} j$.

\section{Condiciones iniciales y límite}

Al inicio del proceso de secado la temperatura y el contenido de humedad del material son uniformes. Las condiciones iniciales son entonces:

$$
\begin{aligned}
& \left.T\right|_{x,=0}=T_{0} \\
& \left.W\right|_{x, t=0}=W_{0}
\end{aligned}
$$

Debido a la simetría, no habrá un gradiente de humedad ni de temperatura en el centro del material $(X=0)$, por lo cual las condiciones límites en el centro son:

$$
\begin{aligned}
& \frac{\partial M}{\left.\partial n\right|_{n=0, t}}=0 \\
& \frac{\partial T}{\left.\partial n\right|_{n=0, t}}=0
\end{aligned}
$$

En la superficie del material, la transferencia de calor ocurre por convección, entonces podemos escribir:

$$
h_{T}\left(T_{\infty}-T_{w b}\right)-k \frac{\partial T}{\partial n}-j\left[h_{\text {vap }}+c p_{\text {agua }}\left(T_{\infty}-T_{s}\right)\right]=0
$$

El principio general de la convección en el secado es el siguiente: un flujo de calor superficial es debido a la diferencia que existe entre la temperatura del medio secante y la temperatura de bulbo húmedo $\left(T_{\infty}-T_{w b}\right)$. El flujo de calor que sale de la superficie de la tabla es cuantificado por la conducción de calor en el producto y además por la energía interna del flux de humedad $j$ que sale de la superficie. Cierta cantidad de calor será absorbida por el flujo de humedad. La ecuación 9 es aplicable cuando la humedad que está en la superficie de la tabla de madera no ha alcanzado su punto de evaporación y está todavía en forma líquida. Una vez que la humedad en la superficie de la madera es evaporada, el término de calor latente de evaporización del agua hvap ${ }_{a g u a}$, es eliminado y la condición límite en la superficie es escrita como:

$$
h_{T}\left(T_{\infty}-T_{w b}\right)-k \frac{\partial \theta}{\partial n} T-j \cdot C p_{\text {agua }}\left(T_{\infty}-T_{s}\right)=0(9)
$$


La transferencia de masa en la superficie ocurre por convección, la representa la expresión

$$
\frac{\partial \theta}{\partial n}
$$

que indica que el flujo de masa es normal a la superficie. La condición límite para la transferencia de masa convectiva en una superficie plana es:

$$
D_{\text {humedad }} \frac{\partial\left(\rho_{\text {agua }} W\right)}{\partial n}=h_{m} \rho_{\text {agua }}\left(W_{e}-W_{\infty}\right)
$$

en donde $h_{m}$ es el coeficiente de transferencia convectivo y $W_{e}$ es el contenido de humedad de equilibrio del producto bajo las condiciones específicas del aire (temperatura y humedad relativa, principalmente), asumiendo que el líquido y el vapor están en equilibrio en la superficie. Estas condiciones implican que la rapidez de eliminación de humedad debido a la convección es igual a la rapidez de flujo de humedad debido a la difusión. Esta condición límite se aplica al aire de secado, ya que el gradiente de contenido de humedad entre la superficie y el agente de secado es la fuerza motriz para la remoción de humedad del producto.

\section{Modelo mecanístico para el transporte de masa en medio poroso}

De acuerdo a $\mathrm{Ni}$ et al. (1999), en teoría de secado, además de la proposición fenomenológica de Luikov, Whitaker en 1977 usó un modelo mecanístico y desarrolló un grupo de ecuaciones para los medios porosos. Whitaker fundamentó su modelo en las ecuaciones de conservación de masa y calor para cada fase presente en el material (sólido, líquido y vapor) y después de un promedio volumen de las fases, obtiene ecuaciones de continuidad y flux para el transporte de masa y calor. El éxito de Whitaker (1977) fue el haber realizado un análisis riguroso de cómo transitar un sistema de un nivel microscópico a un nivel macroscópico con bases convincentes. Las principales consideraciones en el desarrollo de Whitaker son un equilibrio termodinámico local, la aplicación de la ley de Darcy, la ley de Fick, un flujo de infiltración para el transporte de gas, un flujo capilar para el transporte de líquido, una estructura rígida del material (no hay encogimiento) y ausencia de agua ligada. La gran ventaja del modelo mecanístico es que la física del modelo es mejor entendida, las hipótesis son muy claras y los parámetros son bien definidos.

Se toma como hipótesis que existe un equilibrio termodinámico local, por lo cual las temperaturas promedio de las fases (sólida, líquida y gas) son iguales:

$$
\bar{T}_{s}^{s}=\bar{T}_{g}^{g}=\bar{T}_{l}^{l}=\bar{T}
$$

La presión de vapor en el medio es expresada como:

$$
\bar{P}_{v}^{g}=a w \cdot P v_{s a t}
$$

$$
\begin{aligned}
P v_{\text {sat }}= & \exp \left(46.784-\frac{6435}{T+273.15} .\right. \\
& -3.868 \ln (T+273.15))
\end{aligned}
$$

\section{Transferencia de masa}

A continuación se escriben las expresiones para la rapidez de transporte de las diferentes fases. 
Para el líquido:

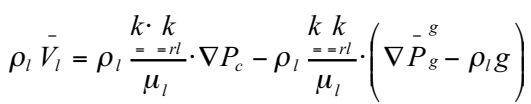

Para el vapor:

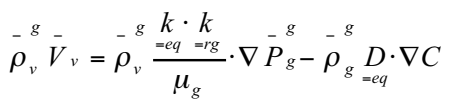

Para el aire:

$$
\bar{\rho}_{a}^{g} \bar{V}_{a}=\bar{\rho}_{a}^{g} \frac{\underset{z e q=r g}{k} \cdot k}{\mu_{g}} \cdot \nabla P_{g}^{g}-\rho_{g}^{g} \underset{=e q}{D} \cdot \nabla C
$$

La difusión-sorción toma la forma fenomenológica de un flux de agua ligada, la cual ha sido discutida abundantemente en la literatura (Stamm, 1946; Stamm, 1960; Stanish et al., 1986):

$$
J_{b}=-\bar{\rho}_{s} D \cdot \nabla W_{b}-\bar{\rho}_{s} \underset{=b t}{D} \nabla \bar{T}
$$

\section{Tranferencia de la fase gaseosa}

A continuación se escriben las ecuaciones para el transporte de la fase gaseosa (Shukla, 1990):

Flujo viscoso de tipo Darcy:

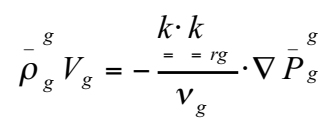

Difusión intermolecular de acuerdo a la ley de Fick

$$
\begin{gathered}
J_{v}=-\bar{\rho}_{g}^{g} D_{=v}^{e f f} \cdot \nabla C \\
\underset{=v}{D^{e f f}}=D_{v} B
\end{gathered}
$$

Se puede calcular un coeficiente de difusión de acuerdo a la proposición de Fuller, Schettler y Giddings (Poling et al., 2000):

$$
D_{\text {bin }}=\frac{10^{-3} T^{1.75}\left[\frac{M_{\text {agua }}+M_{\text {Aire }}}{M_{\text {agua }} M_{\text {Aire }}}\right]^{1 / 2}}{P\left[\left(\sum v\right)_{\text {agua }}^{1 / 3}+\left(\sum v\right)_{\text {Aire }}^{1 / 3}\right]}
$$

\section{Las ecuaciones de conservación de masa}

El balance de masa se escribe para cada uno de los constituyentes en cada fase:

Para el aire seco y el vapor en la fase gaseosa se tienen:

$$
\begin{gathered}
\frac{\partial \bar{\rho}_{a}}{\partial t}+\nabla \cdot\left(\bar{\rho}_{a}^{g} \bar{V}_{a}\right)=0 \\
\frac{\partial \bar{\rho}_{v}}{\partial t}+\nabla \cdot\left(\bar{\rho}_{v}^{g} \bar{V}_{v}\right)=K+K_{b}
\end{gathered}
$$

Para la fase líquida:

$$
\frac{\partial \bar{\rho}_{l}}{\partial t}+\nabla \cdot\left(\bar{\rho}_{l}^{l} \bar{V}_{l}\right)=-K
$$

Para el agua ligada:

$$
\frac{\partial \bar{\rho}_{b}}{\partial t}+\nabla \cdot J_{b}=-K_{b}
$$

Entonces se puede obtener:

$$
\frac{\partial W}{\partial t}+\nabla \cdot\left[\left(\frac{1}{\rho_{s}}\left(\varrho_{l} V_{l}+\rho_{v}^{g} V_{v}+J_{b}\right)\right)\right]=0
$$


Realizando un balance enegético, de acuerdo con Whitaker (1977), se obtiene:

$$
\frac{\partial \bar{H}}{\partial t}+\nabla \cdot\left\{\begin{array}{l}
\left(\rho_{l} \bar{V}_{l} C p_{l}+J_{b} C p_{l}+\bar{\rho}_{a}^{g} \bar{V}_{a} C p_{a}+\bar{\rho}_{v}^{g} \bar{V}_{v} C p_{v}\right) \bar{T} \\
+h_{v}^{0} \bar{\rho}_{v}^{g} \bar{V}_{v}+h_{b}\left(\rho_{l} \bar{V}_{l}+\bar{\rho}_{v}^{g} \bar{V}_{v}\right)-\underset{=}{\lambda \cdot \nabla \bar{T}}
\end{array}\right\}=\Phi
$$

Se establecen las condiciones límites para el modelo mecanístico:

$$
\begin{aligned}
& \Phi=\left(\rho_{l} \bar{V}_{l}+\bar{\rho}_{v}^{g} \bar{V}_{v}+J_{b}\right) \cdot \nabla h_{b} \\
& \left(\frac{1}{\rho_{s}}\left(\rho_{l} \bar{V}_{l}+\rho_{v} \bar{V}_{v}+\overline{\rho_{b} V_{b}}\right)\right) \cdot n=\frac{F_{m}}{\rho_{s}} \\
& \left\{\begin{array}{l}
\left(\rho_{l} \bar{V}_{l} C p_{l}+\overline{\rho_{b} V_{b}} C p_{l}+\bar{\rho}_{a}^{g} \bar{V}_{a} C p_{a}+\bar{\rho}_{v} \bar{V}_{v} C p_{v}\right) \overline{T+} \\
h_{v}^{0} \bar{\rho}_{v}^{g} \bar{V}_{v}+h_{b}\left(\rho_{l} \bar{V}_{l}+\bar{\rho}_{v}^{g} \bar{V}_{v}\right)-\underset{=}{\lambda} \cdot \nabla \bar{T}
\end{array}\right\} \cdot n=Q+\left(h_{v}^{0}+h_{b}+C p_{v} \bar{T}\right) F_{m}
\end{aligned}
$$

La presión capilar $p_{c}$, como lo muestra la figura 1, es una función de la saturación del material (S) y de la temperatura, los efectos de la gravedad son ignorados.

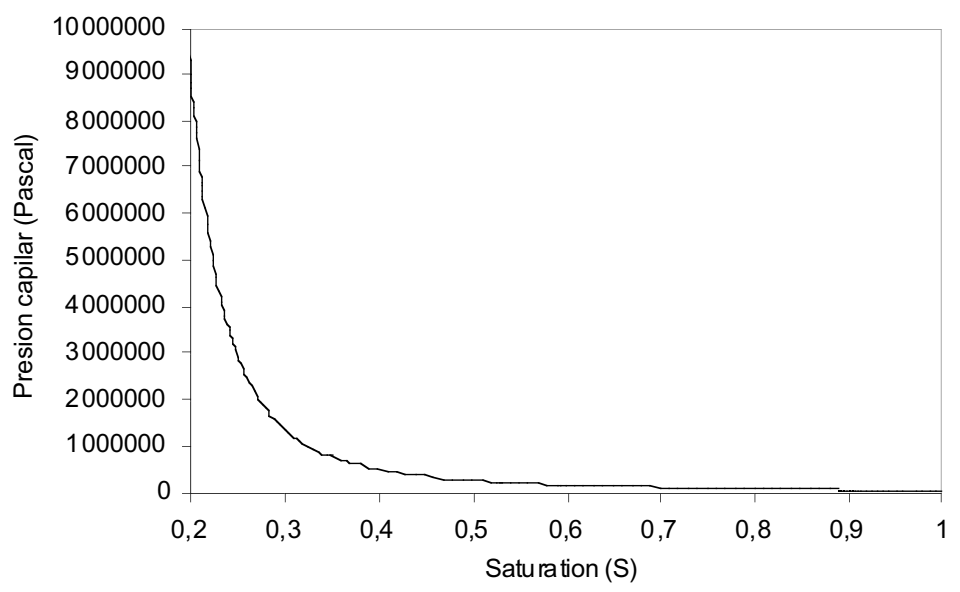

Figura 1. Presión capilar en encino europeo. 
Se considera un contenido de humedad promedio de $0,9 \mathrm{~kg}$ agua $/ \mathrm{kg}$ sólido seco. A continuación se presentan los parámetros que han sido determinados para madera de encino europeo (Hernández (1991):

$$
\begin{gathered}
H R_{e q}=\exp \left(b 1 \cdot b 2^{100 \cdot W}\right) \\
b 1=2.51 \times 10^{-4} \cdot T^{2}-0.1811 \cdot T+35.719 \\
b 2=-9.475 \times 10^{-4} \cdot T+1.133
\end{gathered}
$$

Para la densidad básica de la madera:

$$
\rho(W)=\rho_{\text {madera }}(0.626 W+1)
$$

Presión capilar:

$$
P_{c}=1.5628 \times 10^{3}(1-S) \exp \left(\frac{2.947}{S}\right)
$$

Coeficiente de difusión en el dominio higroscópico:

$$
D_{h}=\frac{1}{\rho_{\text {madera }}} \exp \left[\left(\frac{A_{1}}{T}+A_{2}\right) W+\left(\frac{B_{1}}{T}+B_{2}\right)\right]
$$

$A_{l}=10886.472$

$A_{2}=-31.232$

$B_{1}=-7600.705$

$B_{2}=6.697$

\section{Soluciones analíticas y numéricas}

Las soluciones analíticas pueden ser rigurosas o aproximadas. Las soluciones analíticas rigurosas son solamente para problemas limitados como la ecuación de difusión simple con coeficientes constantes y geometrías regulares; algunos utilizan las transformaciones de Boltzman (Diaw et al., 2006) y de Laplace (Trofimov et al., 1992). Cuando la difusividad es una función de la humedad, lo cual puede ser en el caso del secado de la madera, las soluciones rigurosas no son viables. Para resolver una ecuación de difusión, la difusividad debe ser expresada en ciertas formas, por ejemplo como una expresión exponencial. Las aproximaciones adicionales son necesarias generalmente para simplificar las integrales.

Trabajos recientes como el de Babaszak y Kowalski (1999), presentan soluciones para la deformación de materiales durante el secado haciendo uso de las transformadas de Laplace y la separación de variables. Las soluciones analíticas poseen fuertes requerimientos para la formulación matemática, incluyendo ecuaciones diferenciales, coeficientes, términos fuente, condiciones límite y condiciones iniciales.

En el caso de soluciones numéricas, es común en problemas de transporte de masa y calor, hacer uso de los métodos de elemento finito, volumen finito y diferencias finitas. El problema de modelar mecanismos en bioproductos, como el secado de madera, es que la solución depende de las propiedades, pero también de un fuerte acoplamiento de los procesos, dichos acoplamientos son en general no lineales. Ecuaciones acopladas de transferencia de masa y calor pueden ser resueltas utilizando esquemas iterativos o soluciones directas, dependiendo de la memoria disponible en el computador. Con respecto a la representación gráfica de resultados numéricos, en la actualidad es común el uso de programas comerciales que resuelven ecuaciones diferenciales, o que calculan flujos en medios porosos, por ejemplo FLUEN Inc., AEA-CFDS Inc., MATHCAD y MATLAB. Kocaefe et al. (2006), utilizan FEMLAB para simular el secado de madera aspen a alta temperatura. 


\section{RESULTADOS Y CONCLUSIONES}

En esta última parte se presentan los perfiles de contenido de humedad y de temperaturas para una tabla de madera de una pulgada de espesor, utilizando el modelo mecanístico utilizando mathcad. La madera es sometida a secado convectivo a una temperatura de bulbo seco de $80^{\circ} \mathrm{C}$, una humedad relativa de $30 \%$ y velocidad de flujo de aire de $3 \mathrm{~m} / \mathrm{s}$. Con respecto a la parte de resolución del problema numérico, se utiliza el método implícito en diferencias finitas para discretizar el sistema de ecuaciones diferenciales. Para resolver eficientemente el sistema de ecuaciones resultante, se aplica la factorización LU y el método Crank Nicholson para evitar problemas de inestabilidad (Baehr y Stephan, 1998). La finalidad de esta sección es mostrar al lector, de forma gráfica, que en la actualidad se pueden estimar los perfiles de humedad y temperatura en sólidos, considerando un modelo de secado completo, tomando en cuenta los fenómenos de transporte mencionados anteriormente.
En las figuras 2, 3, 4, y 5 se observan los perfiles de distribución de humedad en una tabla de madera para diferentes tiempos de secado. Como es de esperarse, los perfiles de humedad en general serán parabólicos, el transporte es lento, pues por una parte, la permeabilidad en maderas duras como el encino retrasan el transporte de humedad en el material, y por otra, la remoción de humedad en el dominio higroscópico es lento, es decir, por abajo del punto de saturación.

La figura 6 muestra los perfiles de temperatura en la tabla; en dicha figura se puede observar que la temperatura de la superficie es menor que la temperatura del aire $\left(80^{\circ} \mathrm{C}\right)$ durante casi todo el proceso debido a la evaporación existente en la superficie del material; la evaporación provoca un enfriamiento de la superficie. Sin embargo, al final del proceso, es decir, cuando la tasa de evaporación es casi cero, la temperatura de la pieza será casi igual a la del aire, pues la cantidad de calor cedido al agua es menor, pues es menor la cantidad de masa de agua evaporada.

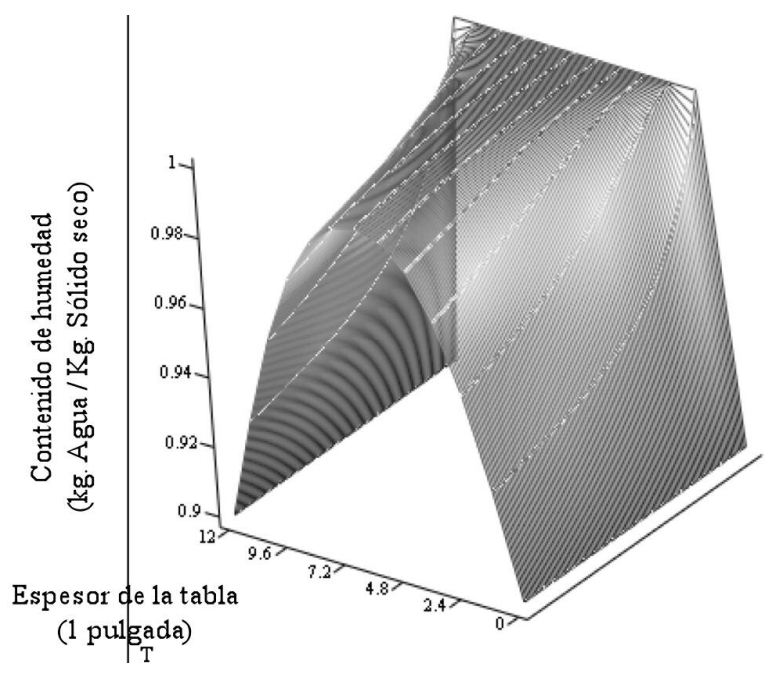

Figura 2. Distribución de humedad en la tabla (2 horas). 


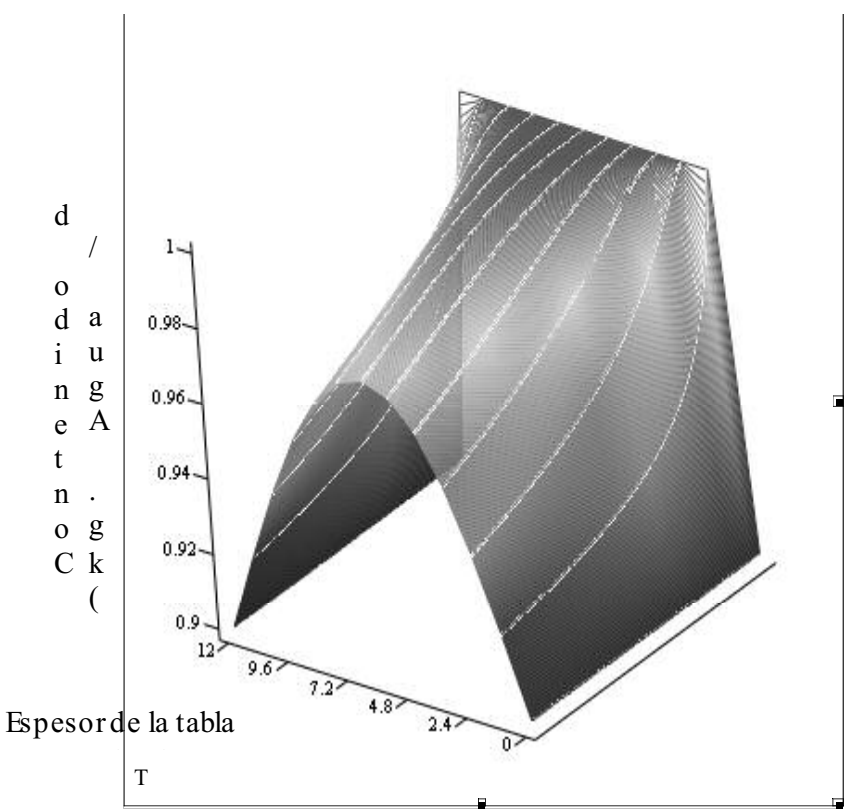

Figura 3. Distribución de humedad en la tabla (10 horas).

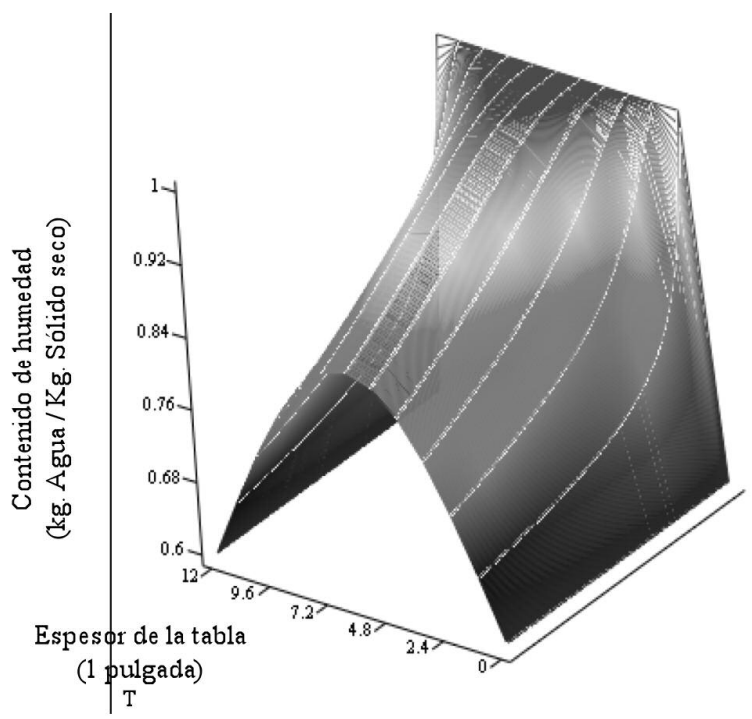

Figura 4. Distribución de humedad en la tabla (48 horas). 


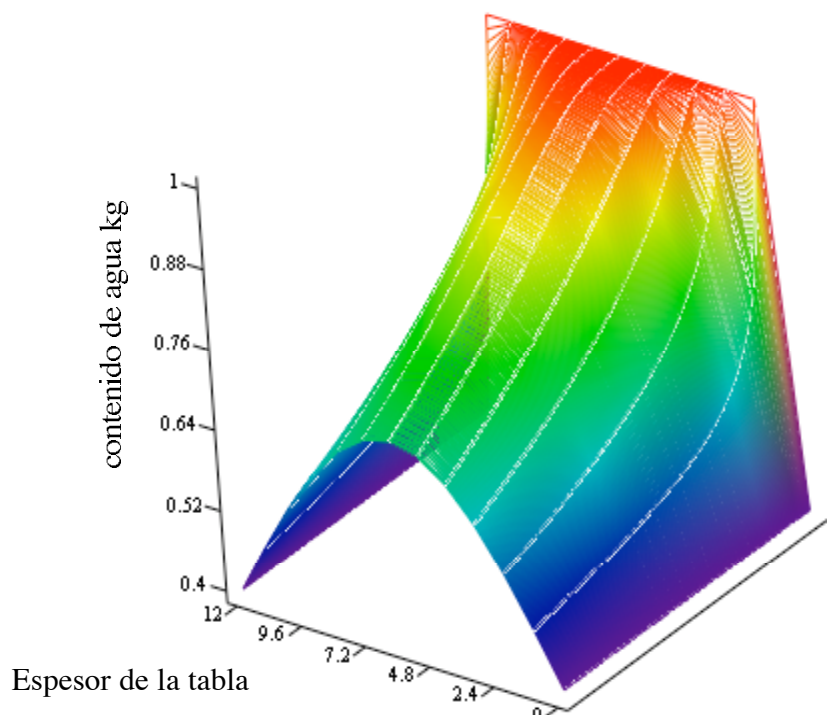

Figura 5. Distribución de humedad en la tabla (100 horas).

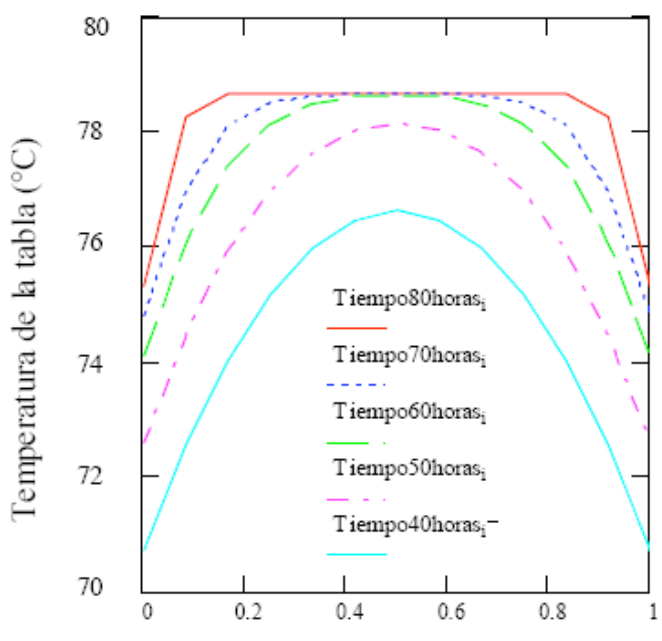

Espesor de la tabla adimensional

Figura 6. Perfil de temperaturas en la tabla de madera. 
La importancia práctica de predecir la distribución de humedad y temperatura en la madera, es conocer la manera en que se desarrollan los gradientes al interior del material, además de predecir en qué momento la diferencia de contenido de humedad entre el centro y la superficie es del $3 \%$, pues de esta forma se reducen los esfuerzos de tensión que se generan, minimizando los riesgos de colapso, de apanalamiento (grietas internas) y de las grietas y rajaduras superficiales. Al término de un proceso de secado, la presencia de esfuerzos debidos a los grandes gradientes de humedad al interior del material o en la superficie, pueden generar defectos de secado como son los colapsos, las rajaduras y torceduras, que disminuyen el valor comercial de las maderas.

Con respecto a la temperatura, la importancia de predecir los perfiles de transferencia de calor, es que en general, en el secado de materiales biológicos, se debe respetar una temperatura máxima en todo el material, de otra forma se corre el riesgo de inducir cambios en su estructura física o bien degradar determinada composición química que es deseable en el producto final; de esta forma se puede tener una idea más clara del momento en que el centro del material ha alcanzado los requerimientos energéticos impuestos. En este caso, el perfil de temperatura presentado en la figura 6 , muestra cómo se desarrollan los gradientes térmicos al interior de la madera. Esto es útil para optimizar los consumos energéticos del proceso; de esta forma se puede saber en qué etapa del secado se puede suministrar menor temperatura, o hacer cambio de condiciones del aire, ya que en la práctica del secado las operaciones industriales son conducidas por programas o secuelas de secado, que son una serie de etapas de proceso, y en cada etapa las condiciones de secado son diferentes.

\section{Notación}

\begin{tabular}{ll}
\hline$D_{=v}^{\text {eff }}$ & Coeficiente de difusión efectivo $\left(\mathrm{m}^{2} / \mathrm{s}\right)$ \\
$D_{v}$ & Coeficiente de difusión de vapor en el aire $\left(\mathrm{m}^{2} / \mathrm{s}\right)$ \\
$k_{\infty}$ & Permeabilidad intrínseca \\
$\rho_{g}^{v}$ & Densidad del vapor en la fase gaseosa $\left(\mathrm{kg} / \mathrm{m}^{3}\right)$ \\
$\rho_{V}^{g}$ & Densidad del aire en la fase gaseosa $\left(\mathrm{kg} / \mathrm{m}^{3}\right)$ \\
$D_{=q}$ & Difusividad equivalente ( $\left.\mathrm{m}^{2} / \mathrm{s}\right)$ \\
$k_{l}$ & Permeabilidad relativa al líquido \\
$\underline{k}_{g}$ & Permeabilidad relativa al gas \\
$P_{V}^{-g}$ & Presión promedio del vapor en el gas $(\mathrm{Pa})$ \\
$D_{V}^{\text {eff }}$ & Coeficiente de Difusión efectivo del vapor $\left(\mathrm{m}^{2} / \mathrm{s}\right)$ \\
$\varepsilon$ & Porosidad \\
$\mu_{\text {air }}$ & Viscosidad del agua líquida (Pa s) \\
$\mu_{g}$ & Viscosidad de la fase gaseosa $(\mathrm{Pa} \mathrm{s})$ \\
$v_{g}$ & Viscosidad cinemática del gas \\
$\rho_{l}$ & Densidad del líquido $\left(\mathrm{kg} / \mathrm{m}^{3}\right)$ \\
$\rho_{\text {madera }}$ & Densidad básica de la madera $\left(\mathrm{kg} / \mathrm{m}^{3}\right)$
\end{tabular}


$v \quad$ Volúmenes de difusión

$a_{w} \quad$ Actividad del agua

$B \quad$ Factor freno

C Concentración de vapor en el aire ( $\mathrm{kg}$ vapor agua/ $\mathrm{kg}$ aire seco)

$C p_{\text {agua }} \quad$ Calor específico del agua $\left(\mathrm{J} / \mathrm{kg}{ }^{\circ} \mathrm{C}\right)$

$\mathrm{Cp}_{\text {madera }} \quad$ Calor específico de la madera $\left(\mathrm{J} / \mathrm{kg}{ }^{\circ} \mathrm{C}\right)$

$D_{b} \quad$ Coeficiente de difusión del agua ligada $\left(\mathrm{m}^{2} / \mathrm{s}\right)$

$D_{b i n} \quad$ Coeficiente de difusión binario $\left(\mathrm{m}^{2} / \mathrm{s}\right)$

$D_{h} \quad$ Coeficiente de difusión en el dominio higroscópico

$D_{\text {humedad }}$ Coeficiente de difusión del agua $\left(\mathrm{m}^{2} / \mathrm{s}\right)$

$G \quad$ Aceleración de la gravedad $(\mathrm{m} / \mathrm{s} 2)$

$h_{T} \quad$ Coeficiente de transferencia de calor (watt $/ \mathrm{m}^{2}{ }^{\circ} \mathrm{C}$ )

$h_{m} \quad$ Coeficiente de transferencia de masa $(\mathrm{m} / \mathrm{s})$

$H R_{e q} \quad$ Humedad relativa de equilibrio (\%)

$h v_{\text {agua }} \quad$ Calor latente de evaporación del agua $(\mathrm{J} / \mathrm{kg})$

$J \quad$ Flujo másico de humedad $\left(\mathrm{kg} / \mathrm{m}^{2} \mathrm{sec}\right)$

$j_{b} \quad$ Flujo másico de agua ligada $\left(\mathrm{kg} / \mathrm{m}^{2} \mathrm{sec}\right)$

$K \quad$ Tasa de evaporación del agua libre (kg agua libre/sec)

$K_{b} \quad$ Tasa de evaporación del agua ligada ( $\mathrm{kg}$ agua ligada/sec)

$k_{\text {madera }} \quad$ Conductividad térmica de la madera (watt $/ \mathrm{m}{ }^{\circ} \mathrm{C}$ )

Magua Peso molecular del agua ( $\mathrm{mol} / \mathrm{g} \mathrm{mol}$ )

$M_{\text {Aire }} \quad$ Peso molecular del aire $(\mathrm{mol} / \mathrm{g} \mathrm{mol})$

$n$ Normal

$P \quad$ Presión del sistema $(\mathrm{Pa})$

$P c \quad$ Presión capilar $(\mathrm{Pa})$

$P v_{\text {sat }} \quad$ Presión de vapor saturado $(\mathrm{Pa})$

$R \quad$ Constante de los gases ideales

$S \quad$ Saturación líquida

$t \quad$ Tiempo (sec)

$T_{\infty} \quad$ Temperatura del aire $\left({ }^{\circ} \mathrm{C}\right)$

$T_{\text {madera }} \quad$ Temperatura promedio de la madera $\left({ }^{\circ} \mathrm{C}\right)$

$T_{S} \quad$ Temperatura superficial del sólido $\left({ }^{\circ} \mathrm{C}\right)$

$T_{w b} \quad$ Temperatura de bulbo húmedo $\left({ }^{\circ} \mathrm{C}\right)$

$W \quad$ Contenido de humedad de la madera (kg agua/kg madera seca)

$W_{\infty} \quad$ Contenido de humedad crítico ( $\mathrm{kg}$ agua/kg madera seca)

$W_{e} \quad$ Contenido de humedad de equilibrio (kg agua/kg madera seca)

$W_{p s f} \quad$ Punto de saturación de la fibra (kg agua/kg madera seca) 


\section{AGRADECIMIENTOS}

Se agradece sinceramente al CONACYT por la beca de estudios otorgada al autor para la realización de sus estudios de Doctorado en Francia.

\section{REFERENCIAS}

Babaszak, J. y Kowalski, S. J. 1999. Stresses in viscoelastic plate dried convectively, Drying Technology 17: $97-117$

Baehr, H.D. y K. Stephan.1998. Heat and Mass Transfer. 2a. ed.,. SpringerVerlag. New York. 671 p.

Bird, R.B., Stewart, W.E. y Lightfoot, E.N. 2006. Transport phenomena. 2a. ed.,. John Wiley. 920 p.

Diaw, M., Perrin, B. y Monlouis-Bonnaire, J-P. 2006. Limit of validity of the moisture diffusivity for the study of moisture transfer inside terracotta. Materials and Structures 35(1): 42-49.

Esfahanian. A. H. 1999. A numerical modelling study of transport phenomena in wood drying. PhD-Thesis at the University of Victoria. Mechanical Engineering.

Incropera, F. P., DeWitt, D. P., Bergman, T. L. y Lavine, A. S. 2006. Fundamentals of Heat and Mass Transfer. 6a. ed., Wiley. 1024 p.

Geankoplis, C. 2003. Transport Processes and Separation Process Principles. 4a. ed., Prentice Hall PTR. 1056 p.

Hernández, J. M. 1991. Secado de Encino. Caracterización, proceso por convección y en vacío. PhDThesis Universidad de Burdeos.
Keey, R., T. Langrish y J.C.F. Walker. 2000. Kiln-Drying of Lumber. 1a. ed., Springer Series in Wood Science. $326 \mathrm{p}$.

Kocaefe, D., Younsi, R., Chaudry, B. y Kocaefe, Y. 2006. Modeling of heat and mass transfer during high temperature treatment of aspen. Wood Sci. Technol. Online First.

Majumdar, P. 2005. Computational Methods for Heat and Mass Transfer. 1a. ed., Taylor and Francis. $744 \mathrm{pp}$.

Martinovic, D., Horman, I. y Demirdzic. 2001. Numerical and Experimental analysis of a wood drying process. Wood Science and Technology 35:143-156.

Middleman, D. 1997. An Introduction to Mass and Heat Transfer: Principles of Analysis and Design. 2a. ed.,Wiley. 696 pp.

Ni, H., Datta, A.K. y Torrance, K.E. 1999. Moisture transport in intensive microwave heating of biomaterials: a multiphase porous media model. Int. Journal of Heat and Mass transfer, 42:1501-1512.

Perré, P. y I.W. Turner. 2002. A heterogeneous wood drying computational model that accounts for material property variation across growth rings. Chemical Engineering Journal 86:117-131.

Poling, B. E., Prausnitz, J. y O'Connell, J. 2000. The properties of gases and Liquids. 5a. d., McGrawhill Professional. $768 \mathrm{pp}$.

Shukla, K. N. 1990. Diffusion Processes During Drying of Solids (Series in Theoretical and Applied Mechanics, 
Vol 11. World Scientific Publishing Company. Londres. 168 p.

Siau, J. F. 1984. Transport Process in Wood. Springer Verlag. Germany. $245 \mathrm{p}$.

Stamm, A. J. Passage of liquids, vapors and dissolved materials through softwoods. US. Dept. Agric. Techn-Ball. Nr. 929, Washington DC. Oct. 1946.

Stamm, A. J. 1960. Combined boundwater and water vapour diffusion into Sitka Spruce. Forest Prod. J. 10:644-648.

Stanisch, M. A., Schajer, G.S. y Kayihan, F. 1986. A mathematical model of Drying for hygroscopic porous media. AIChE J. 32(8):1301-1311.

Trofimov, A. S., Kryzhnii, V. V. y Kryzhnyaya, E. P. 1992. Inverse boundary value problem of a drying process. Journal of Engineering physics and Thermophysics 67(1-2): 123-126.
Truscott, S.L. y Turner, I.W. 2006. A heterogeneous three-dimensional computational model for wood drying. Applied Mathematical Modelling. 29(4):381-410.

Turner, I. W. y P. Perré. 2001. The use of implicit flux limiting schemes in the simulation of drying process: A new maximum flow sensor applied to phase mobilities. Applied mathematical modelling 25:513-540.

Williamson T.G. 2001. APA Engineered Wood Handbook. 1a. ed., McGrawHill. 750 p. 15(1):75-89. 
Página en blanco intencionalmente (This page left blank intentionally) 